\section{OPEN ACCESS}

Edited by:

Anca Dana Dobrian,

Eastern Virginia Medical School,

United States

Reviewed by:

Zhichao Feng,

Albert Einstein College of Medicine,

United States

Akinobu Nakamura,

Hokkaido University, Japan

${ }^{*}$ Correspondence:

Xiao Qun Wang

xiaoqun_wang@hotmail.com

Lin Lu

rilulin1965@163.com

${ }^{\dagger}$ These authors have contributed equally to this work

Specialty section: This article was submitted to Diabetes,

a section of the journal

Frontiers in Endocrinology

Received: 24 October 2018 Accepted: 07 March 2019

Published: 04 April 2019

Citation:

Chen JW, Li C, Liu ZH, Shen Y, Ding $F H$, Shu $X Y$, Zhang RY, Shen WF, Lu L and Wang XQ (2019) The Role of Monocyte to High-Density Lipoprotein

Cholesterol Ratio in Prediction of

Carotid Intima-Media Thickness in

Patients With Type 2 Diabetes.

Front. Endocrinol. 10:191 doi: 10.3389/fendo.2019.00191

\title{
The Role of Monocyte to
} High-Density Lipoprotein Cholesterol Ratio in Prediction of Carotid Intima-Media Thickness in Patients With Type 2 Diabetes

Jia Wei Chen ${ }^{1,2+}$, Chang $\mathrm{Li}^{1,2+}$, Zhu Hui Liu ${ }^{1}$, Ying Shen ${ }^{1}$, Feng Hua Ding ${ }^{1}$, Xin Yi Shu ${ }^{1}$,
Background: Chronic inflammatory disorders and dyslipidemia in type 2 diabetes mellitus (T2DM) are essential contributors to the development of atherosclerotic cardiovascular disease. Monocyte to high-density lipoprotein cholesterol (HDL-C) ratio $(\mathrm{MHR})$ is a novel and simple measure associated positively with the body inflammatory and oxidative stress status. However, little is known regarding the role of $\mathrm{MHR}$ in evaluating carotid intima-media thickness (CIMT), a surrogate predictor of subsequent vascular events, especially in diabetic patients.

Methods: A total of 494 patients with T2DM and 1,848 non-diabetic subjects were consecutively enrolled in study 1. Correlation between MHR and CIMT was compared between diabetic and non-diabetic subjects. In study 2, a total of 110 T2DM patients from study 1 with normal basal CIMT and a follow-up ultrasonography at 12 months were enrolled. The predictive role of MHR on CIMT progression in diabetic patients was analyzed.

Results: In study 1, MHR was higher in patients with T2DM than non-diabetic subjects $(p<0.001)$. After adjustment for confounding risk factors, MHR remained correlated significantly with CIMT in diabetic $(r=0.172, p=0.001)$ but not non-diabetic $(r=0.006$, $p=0.813$ ) subjects. Logistic regression analyses demonstrated that MHR is superior to traditional lipid parameters in association with elevated CIMT in diabetic patients. In study 2, MHR at baseline was positively correlated with change in CIMT $(r=0.313$, $p=0.001$ ). Basal MHR was independently associated with change in CIMT $[\beta=0.059$, (95\% Cl: 0.012-0.105), $p=0.014$ ] in multivariate linear regression analysis.

Conclusions: Our study suggests that $\mathrm{MHR}$ is a convenient and effective measure in prediction of the presence and progression of subclinical carotid atherosclerosis in patients with T2DM.

Keywords: monocyte to high-density lipoprotein cholesterol ratio, carotid intima-media thickness, atherosclerosis, type 2 diabetes, subclinical carotid atherosclerosis 


\section{INTRODUCTION}

Patients with type 2 diabetes mellitus (T2DM) are predisposed to develop atherosclerosis, which is largely attributable to the disturbed glucose and lipid metabolism as well as the chronic inflammatory status $(1,2)$. The compositional changes of lipoprotein particles in diabetic conditions, as characterized by the formation of atherogenic small dense low-density lipoprotein (LDL) particles and the predominance of large very-low density lipoprotein (VLDL) particles, usually lead to underappreciation of the risk associated with the atherogenic lipoprotein by simply measuring the cholesterol content in LDL (LDL-C) (3). On the

\section{Study 1}

Consecutive diabetic and non-diabetic subjects

(2014/6-2016/9, n=2711)

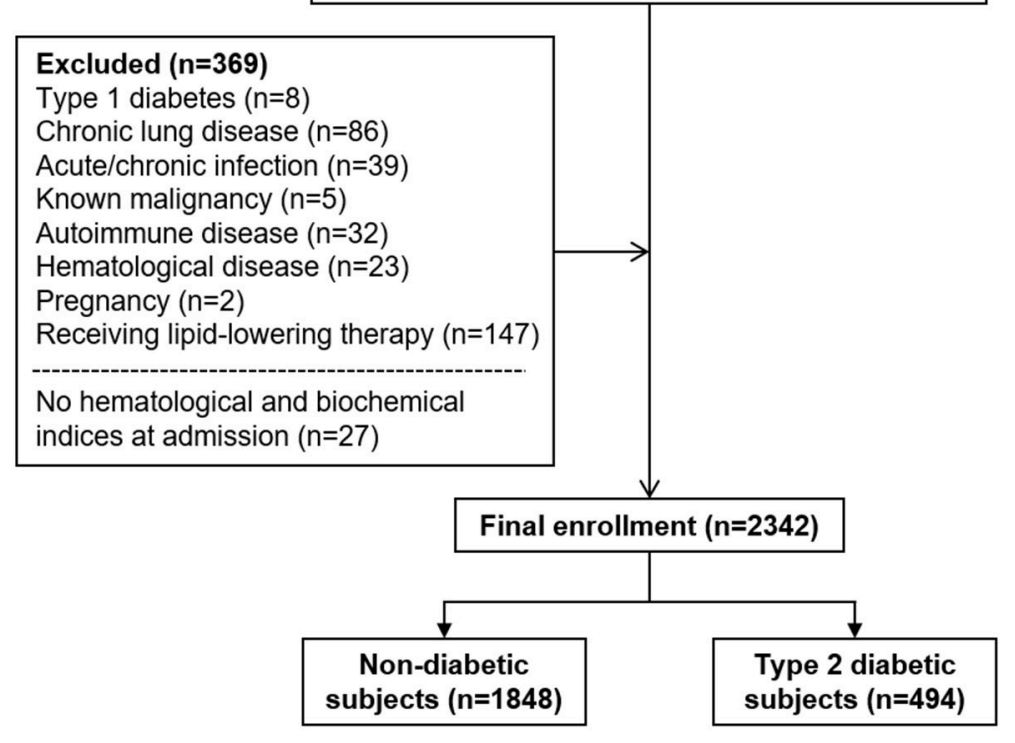

\section{Study2}

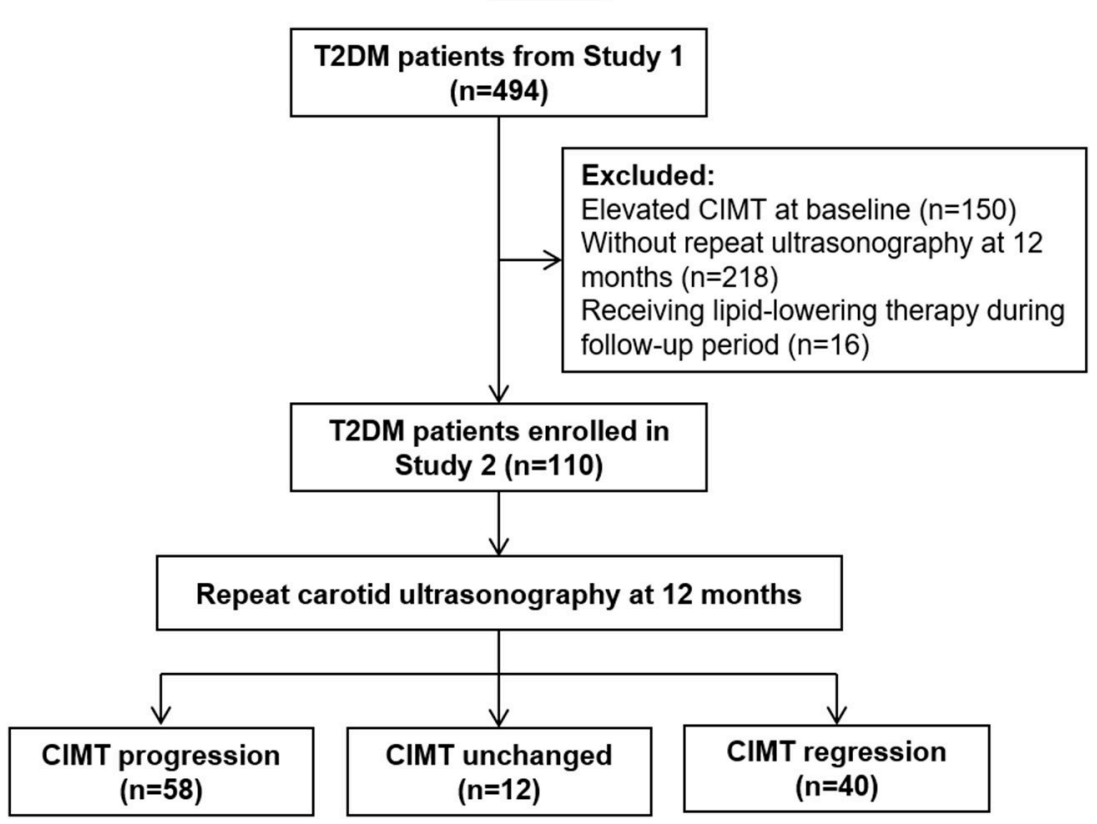

FIGURE 1 | Flow charts of patient enrollment in Study 1 and Study 2. 
other hand, chronic inflammation plays a comparable role in driving atherosclerosis in diabetic conditions $(4,5)$. Monocyte is one of the circulating makers of systemic inflammation and a fundamental player in atherogenesis (6). The entire process of monocytosis, adhesion, and infiltration of monocytes to the vessel wall, and the subsequent transformation into lipid-laden macrophages has been well-described in human and animal studies $(7,8)$. Several lines of evidence have shown that count of monocytes or monocyte subsets are independent predictors of subclinical atherosclerosis or coronary artery disease $(9,10)$.

Monocyte to HDL cholesterol ratio (MHR) was recently defined as a novel marker in relation to the extent of inflammation and oxidative stress as well as adverse cardiovascular outcomes (11). Previous reports have shown that MHR is associated with cardiovascular events in patients with chronic kidney disease (12), and in-hospital and long-term death in patients with infective endocarditis and normal left ventricular function (13).
Carotid intima-media thickness (CIMT) is a simple and costeffective surrogate phenotype of subclinical atherosclerosis. A number of longitudinal studies have evidenced the predictive value of CIMT for subsequent cardiac and cerebrovascular events $(14,15)$. In the present study, we investigated the association of MHR with CIMT in diabetic and non-diabetic subjects. We also analyzed the role of MHR in prediction of CIMT progression by performing carotid ultrasonography at 1 year follow-up in T2DM patients.

\section{METHODS}

\section{Study Population}

This study complies with the Declaration of Helsinki. The study protocol was approved by the local hospital ethics committee, and written informed consent was obtained from all participants.

For the purpose of this study to assess preclinical atherosclerosis, we consecutively enrolled 2,711 subjects in

TABLE 1 | Baseline characteristics of diabetic and non-diabetic subjects.

\begin{tabular}{|c|c|c|c|}
\hline & non-DM & DM & $P$-value \\
\hline Number & 1,848 & 494 & \\
\hline Male gender, $n(\%)$ & $958(51.8)$ & $276(55.9)$ & 0.116 \\
\hline Age, years & $59.73 \pm 8.89$ & $60.71 \pm 9.07$ & 0.030 \\
\hline Body mass index, $\mathrm{kg} / \mathrm{m}^{2}$ & $24.64 \pm 3.38$ & $25.49 \pm 3.71$ & $<0.001$ \\
\hline Smoking, $n(\%)$ & $344(18.6)$ & $94(19.0)$ & 0.845 \\
\hline Hypertension, $n$ (\%) & $942(51.0)$ & $329(66.6)$ & $<0.001$ \\
\hline Systolic blood pressure, $\mathrm{mmHg}$ & $132.28 \pm 17.93$ & $136.57 \pm 18.70$ & $<0.001$ \\
\hline Diastolic blood pressure, mmHg & $76.95 \pm 11.08$ & $76.82 \pm 11.52$ & 0.813 \\
\hline White blood cells $\left(10^{9} / \mathrm{mL}\right)$ & $5.88 \pm 1.40$ & $6.27 \pm 1.62$ & $<0.001$ \\
\hline Neutrophils $\left(10^{9} / \mathrm{mL}\right)$ & $3.37 \pm 1.10$ & $3.60 \pm 1.21$ & $<0.001$ \\
\hline Lymphocytes (109/mL) & $1.87 \pm 0.58$ & $1.99 \pm 0.60$ & $<0.001$ \\
\hline Monocytes $\left(10^{9} / \mathrm{mL}\right)$ & $0.46 \pm 0.14$ & $0.47 \pm 0.14$ & 0.254 \\
\hline Platelets $\left(10^{9} / \mathrm{mL}\right)$ & $183.98 \pm 49.68$ & $184.66 \pm 51.45$ & 0.792 \\
\hline hsCRP, mg/L & $0.73(0.40-1.52)$ & $0.90(0.46-1.93)$ & 0.002 \\
\hline Serum creatinine, $\mu \mathrm{mol} / \mathrm{L}$ & $72.53 \pm 12.12$ & $72.71 \pm 14.21$ & 0.788 \\
\hline Blood urea nitrogen, mmol/L & $5.48 \pm 1.37$ & $5.58 \pm 1.40$ & 0.164 \\
\hline Uric acid, $\mu \mathrm{mol} / \mathrm{L}$ & $327.50 \pm 81.23$ & $319.37 \pm 81.33$ & 0.049 \\
\hline eGFR, $\mathrm{mL} / \mathrm{min} / 1.73 \mathrm{~m}^{2}$ & $111.67 \pm 17.53$ & $114.85 \pm 21.14$ & 0.001 \\
\hline HbA1c, \% & $5.60(5.40-5.90)$ & $6.90(6.30-7.80)$ & $<0.001$ \\
\hline Fasting glucose, mmol/L & $4.97(4.58-5.42)$ & $6.43(5.47-7.77)$ & $<0.001$ \\
\hline Postprandial glucose (2 h), mmol/L & $6.55(5.56-7.61)$ & $13.43(10.36-16.52)$ & $<0.001$ \\
\hline Fasting insulin, $\mu \mathrm{IU} / \mathrm{mL}$ & $8.33(5.75-11.59)$ & $9.49(6.30-14.65)$ & $<0.001$ \\
\hline Postprandial insulin (2 h), $\mu \mathrm{IU} / \mathrm{mL}$ & 46.76 (25.29-77.26) & 47.34 (27.58-79.11) & 0.680 \\
\hline Triglyceride, $\mathrm{mmol} / \mathrm{L}$ & $1.30(0.97-1.83)$ & $1.58(1.10-2.21)$ & $<0.001$ \\
\hline Total cholesterol, mmol/L & $4.33 \pm 1.01$ & $4.32 \pm 1.14$ & 0.766 \\
\hline HDL cholesterol, mmol/L & $1.21 \pm 0.29$ & $1.12 \pm 0.29$ & $<0.001$ \\
\hline LDL cholesterol, mmol/L & $2.58 \pm 0.82$ & $2.55 \pm 0.90$ & 0.471 \\
\hline Apolipoprotein A-I, g/L & $1.31 \pm 0.20$ & $1.28 \pm 0.22$ & 0.004 \\
\hline Apolipoprotein B, g/L & $0.82 \pm 0.22$ & $0.85 \pm 0.24$ & 0.052 \\
\hline $\mathrm{MHR}$ & 9.95 (7.32-12.93) & 10.92 (8.34-14.02) & $<0.001$ \\
\hline Oral hypoglycemic drugs, $n$ (\%) & - & $338(68.4)$ & - \\
\hline Insulin, $n(\%)$ & - & $131(26.5)$ & - \\
\hline
\end{tabular}


study 1 (Figure 1, upper part) based on the following criteria (1) aged between 35 and 80 years old (2), no manifest concomitant atherosclerotic disease, from June, 2014 to September, 2016 in the Department of Cardiology, Rui Jin Hospital, Shanghai Jiao Tong University School of Medicine. To avoid confounding data, we excluded 342 patients due to type 1 diabetes, pregnancy, chronic lung disease, chronic or acute infection, known malignancy, autoimmune or hematologic disease, or receiving lipid-lowering therapy. Another 27 patients who did not have hematological and biochemical indices that included monocyte and HDL-C on admission were also excluded. Thus, 2,342 patients comprised the final enrollment. The diagnosis of diabetes was made according to the criteria of American Diabetes Association (16). Hypertension and dyslipidemia were diagnosed according to seventh report of the Joint National Committee on prevention, detection, evaluation, and treatment of high blood pressure (JNC 7) and guideline of the National Cholesterol Education Program (ATP III), respectively $(17,18)$.

To investigate the predictive role of $\mathrm{MHR}$ on CIMT progression in T2DM patients with relatively normal CIMT, a total of 126 diabetic patients from study 1 with normal CIMT (within the first three quartiles, $<0.73 \mathrm{~mm}$ ) and a follow-up ultrasonography at 12 months were enrolled in study 2 (Figure 1, bottom part). Sixteen patients were excluded due to receiving lipid-lowering therapy within this period. The change in CIMT per year (mm/year) was calculated. The association between change in CIMT and MHR was then analyzed in the final 110 diabetic patients.

TABLE 2 | Correlation analyses for CIMT and MHR in diabetic and non-diabetic subjects.

\begin{tabular}{|c|c|c|c|c|}
\hline & \multicolumn{4}{|c|}{ CIMT } \\
\hline & \multicolumn{2}{|c|}{ non-DM } & \multicolumn{2}{|c|}{ DM } \\
\hline & $r$ & $P$-value & $r$ & $P$-value \\
\hline Age & 0.197 & $<0.001$ & 0.216 & $<0.001$ \\
\hline Systolic BP & 0.080 & 0.001 & 0.070 & 0.120 \\
\hline Diastolic BP & 0.043 & 0.062 & 0.009 & 0.848 \\
\hline $\mathrm{BMl}$ & 0.066 & 0.005 & 0.004 & 0.930 \\
\hline Monocytes & 0.023 & 0.329 & 0.088 & 0.050 \\
\hline Log hsCRP & 0.056 & 0.019 & 0.001 & 0.981 \\
\hline eGFR & -0.044 & 0.069 & -0.130 & 0.005 \\
\hline Log $\mathrm{HbA} 1 \mathrm{C}$ & 0.133 & $<0.001$ & -0.026 & 0.568 \\
\hline Log triglyceride & 0.044 & 0.061 & 0.063 & 0.159 \\
\hline Total cholesterol & 0.043 & 0.065 & 0.008 & 0.862 \\
\hline HDL cholesterol & -0.060 & 0.010 & -0.079 & 0.078 \\
\hline LDL cholesterol & 0.068 & 0.004 & 0.042 & 0.352 \\
\hline non-HDL cholesterol & 0.063 & 0.007 & 0.029 & 0.521 \\
\hline Apolipoprotein A-I & -0.050 & 0.032 & -0.051 & 0.256 \\
\hline Apolipoprotein B & 0.070 & 0.003 & 0.029 & 0.516 \\
\hline Log MHR & 0.058 & 0.012 & 0.126 & 0.005 \\
\hline Log MHR adjusted* & 0.006 & 0.813 & 0.172 & 0.001 \\
\hline
\end{tabular}

${ }^{*}$ After adjustment for age, sex, history of smoking, systolic blood pressure, body mass index, high sensitivity CRP, eGFR, HbA1C, and LDL cholesterol.

\section{Clinical and Biochemical Assessments}

Blood samples were collected after an overnight fasting. Serum glucose, blood urea nitrogen, creatinine, uric acid, total cholesterol, low-density lipoprotein-cholesterol (LDL-C), high-density lipoprotein cholesterol (HDL-C), and triglycerides were assessed (HITACHI 912 Analyzer, Roche Diagnostics, Germany). The estimated glomerular filtration rate (eGFR) was computed using the Chronic Kidney Disease Epidemiology Collaboration equation (19). Blood HbAlc concentration was measured using ion-exchange high performance liquid chromatography with Bio-rad Variant Hemoglobin Testing System (Bio-Rad Laboratories, USA). Serum levels of high sensitive C-reactive protein (hsCRP) were determined by ELISA (Biocheck Laboratories, Toledo, OH, USA). The detailed information about medical history and lifestyles including smoking and drinking status was obtained using a standard questionnaire by the trained physicians. Current smoking status was defined as yes if the subject smoked at least one cigarette per day or seven cigarettes per week in the past 6 months. Body mass index (BMI) was calculated using the formula of weight/height ${ }^{2}$ (kilograms per square meter). Blood pressure was measured on the non-dominant arm in a seated position after a 10-min rest, using an electronic blood pressure monitor (OMRON Model HEM-752 FUZZY' Omron Co., Dalian, China). Three measurements were taken at 1-min intervals, and the average was used for analysis. MHR was calculated by monocyte counts $\left(\times 10^{6} / \mathrm{L}\right) / \mathrm{HDL}-\mathrm{C}(\mathrm{mg} / \mathrm{dL})$.

\section{CIMT Measurements}

CIMT measurements were performed manually using a highresolution B-mode tomographic ultrasound system (Esaote Mylab90, Italy) with a linear $10 \mathrm{MHz}$ transducer. Precision of the CIMT measurement is $0.01 \mathrm{~mm}$. The sonographers measured CIMT on the far-wall of the right and left common carotid arteries, $1.5 \mathrm{~cm}$ proximal to the bifurcation. The transducer was manipulated so that the lumen diameter was maximized in

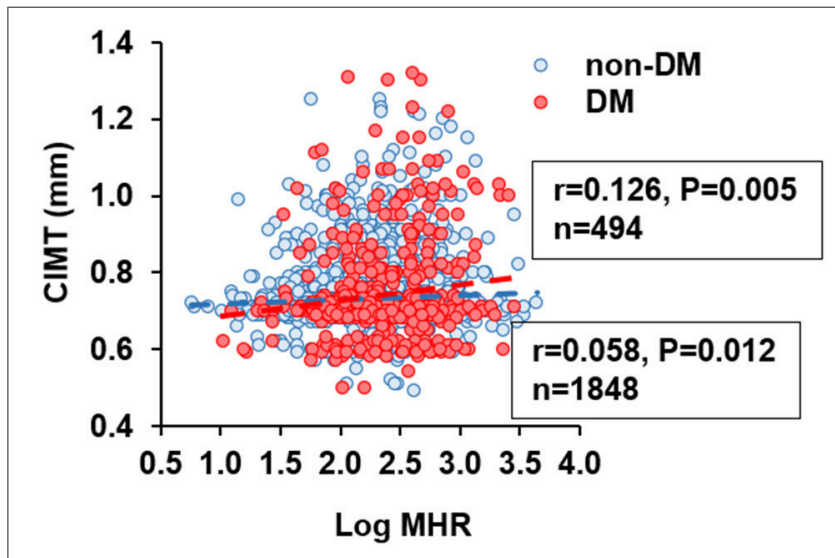

FIGURE 2 | Correlation between carotid intima-media thickness (CIMT) and monocyte to HDL cholesterol ratio (MHR). MHR was logarithmically transformed before plotting. Blue circle and dashed line, non-diabetic subjects ( $n=1848)$; red circle and dashed line, patients with type 2 diabetes $(n=494)$. 
the longitudinal plane. The first and second lines represent the lumen-intimal interface and the collage-contained upper layer of tunic adventitia, respectively. The mean value of the right and left common carotid IMT was used for analysis. The fourth quartile of CIMT ( $\geq 0.73 \mathrm{~mm}$ ) was defined as elevated CIMT. In study 2, the baseline and follow-up CIMT were recorded as CIMT1 and CIMT2, respectively. Progression of CIMT was calculated as the

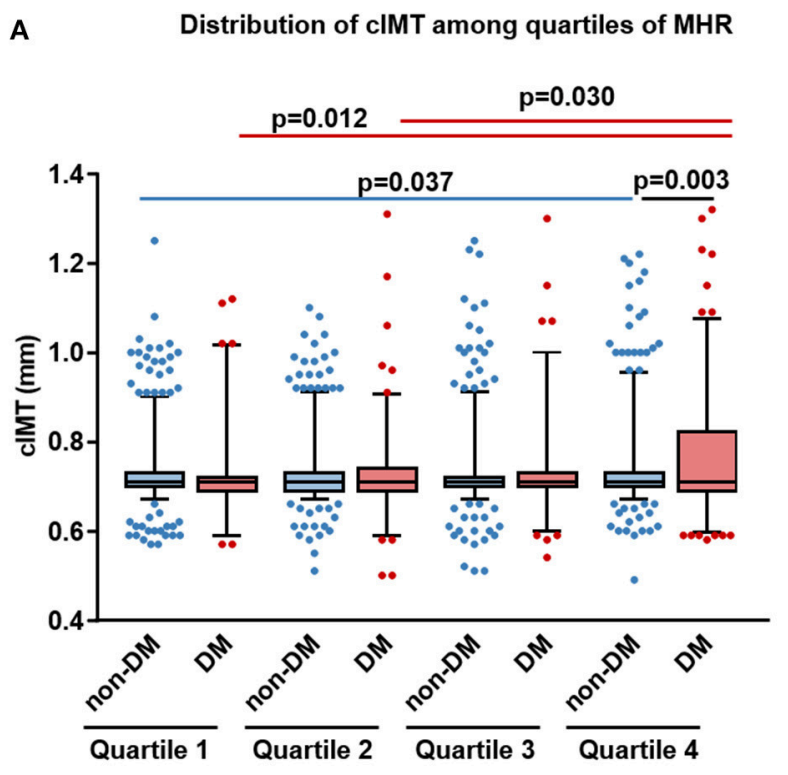

B Distribution of maximal cIMT among quartiles of MHR

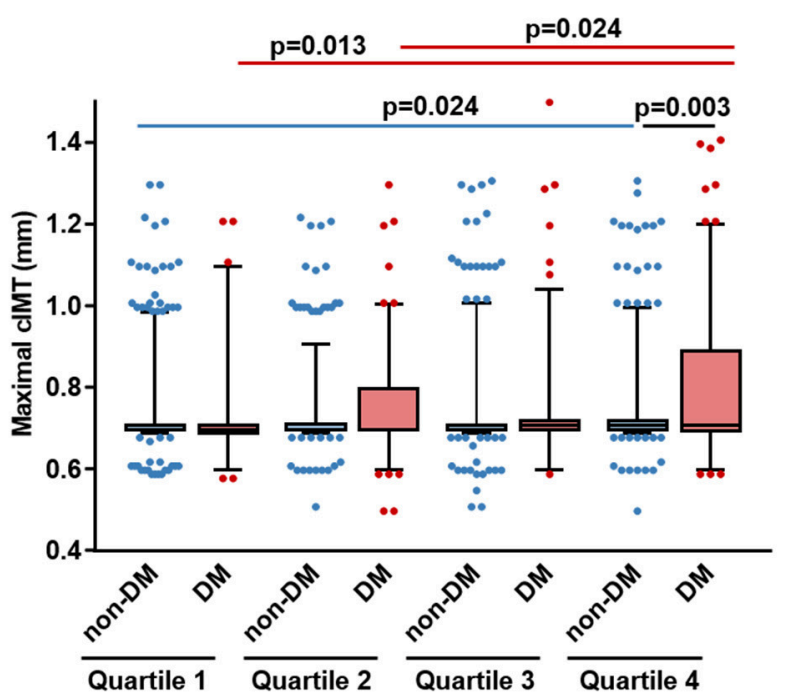

FIGURE 3 | Distribution of average and maximal carotid intima-media thickness (CIMT) among different quartiles of monocyte to HDL cholesterol ratio (MHR) in diabetic and non-diabetic subjects. Box and whisker plots of the average (A) and maximal (B) CIMT in different quartiles of MHR were shown (horizontal bars in box: low, 25 percentile; middle, median; upper, 75 percentile; low whisker, 5 percentile, and upper whisker, 95 percentile). DM, patients with type 2 diabetes; non-DM, non-diabetic patients. difference between CIMT1 and CIMT2 and recorded as change in CIMT. The coefficient of variations was $<5.1 \%$.

\section{Statistics}

Continuous variables were presented as mean (SD or SEM), and categorical data were summarized as frequencies (percentages). For categorical clinical variables, differences between groups were evaluated by the chi-square test followed by Bonferroni's correction. For continuous variables, normal distribution was evaluated with Kolmogorov-Smirnov test, and logarithmic transformations were performed on the continuous variables of non-normal distribution. Differences among groups were analyzed by Student's $t$-test or one-way analysis of variance (ANOVA) followed by post-hoc Bonferroni test. Correlation between variables was determined by Pearson's correlation test. In study 1 , different logistic regression models were implemented to interrogate the association of different lipid parameters with elevated CIMT in patients with T2DM and nondiabetic patients. In model 1 , no covariates were adjusted; in model 2, age, sex, history of hypertension and smoking were adjusted; in model 3, eGFR, logarithmically transformed levels of high sensitive C-reactive protein, $\mathrm{HbAlc}$, and triglyceride were further adjusted. In study 2, linear regression was performed to evaluate the associations between the change in CIMT and MHR in patients with T2DM. Confounders adjusted in the linear regression included baseline CIMT, age, sex, history of hypertension and smoking, logarithmically transformed levels of $\mathrm{HbAlc}$, and triglyceride. All statistical analyses were performed using the SPSS 23.0 for Windows (SPSS, Inc., Chicago, IL, USA). A two-tailed $<0.05$ was considered statistically significant.

\section{RESULTS}

\section{Study 1}

\section{Characteristics of the Studied Population}

A total of 494 patients with T2DM and 1,848 non-diabetic subjects were analyzed in study 1 (Table 1). Compared to nondiabetic subjects, patients with T2DM were of older age and had higher prevalence of hypertension. Counts of total white blood cells, neutrophils, lymphocytes, and levels of high sensitivity Creactive protein (hsCRP), triglyceride were higher, whereas levels of HDL-C and apolipoprotein A-I (apoA-I) were lower in diabetic than non-diabetic patients. No significant difference in levels of total cholesterol, LDL-C, serum creatine, and blood urea nitrogen was detected between two groups. The monocyte to HDL-C ratio (MHR) was higher in T2DM patients than non-diabetic subjects [10.92 (interquartile rage (IQR): 8.34-14.02) vs. 9.95 (IQR: 7.32-12.93), $p<0.001$ ].

\section{Correlation Analyses of CIMT and MHR}

In non-diabetic subjects, we found CIMT was correlated positively with age, systolic blood pressure (BP), BMI, LDL-C, non-HDL cholesterol, apolipoprotein (apoB), log-transformed levels of hsCRP, HbA1C, and MHR ( $r=0.058, p=0.012)$, while negatively with HDL-C and apoA-I. However, most of these associations were attenuated in patients with T2DM, with the 
TABLE 3 | Sex stratified associations of MHR with CIMT in diabetic and non-diabetic patients.

\begin{tabular}{|c|c|c|c|c|c|c|c|c|c|c|c|c|}
\hline & \multicolumn{6}{|c|}{ Model 1} & \multicolumn{6}{|c|}{ Model 2} \\
\hline & \multicolumn{3}{|c|}{ Males } & \multicolumn{3}{|c|}{ Females } & \multicolumn{3}{|c|}{ Males } & \multicolumn{3}{|c|}{ Females } \\
\hline & $\mathbf{S} \beta$ & $R^{2}$ & $P$-value & $\mathbf{S} \beta$ & $R^{2}$ & $P$-value & $\mathbf{S} \beta$ & $R^{2}$ & $P$-value & $\mathbf{S} \beta$ & $R^{2}$ & $P$-value \\
\hline non-DM & 0.071 & 0.062 & 0.024 & 0.011 & 0.027 & 0.737 & 0.029 & 0.083 & 0.416 & -0.006 & 0.040 & 0.878 \\
\hline DM & 0.153 & 0.081 & 0.009 & 0.066 & 0.030 & 0.323 & 0.221 & 0.089 & 0.001 & 0.089 & 0.036 & 0.256 \\
\hline
\end{tabular}

$R^{2}$, adjusted $R$-squared, $S \beta$, standardized $\beta$ coefficient.

Model 1, age-adjusted associations.

Model 2, after adjusting for sex, history of smoking, systolic blood pressure, body mass index, high sensitivity CRP, eGFR, HbA1C, and LDL cholesterol.

TABLE 4 | Logistic regression analyses for elevated CIMT in diabetic and non-diabetic subjects.

\begin{tabular}{|c|c|c|c|c|}
\hline Model & \multicolumn{2}{|c|}{ non-DM } & \multicolumn{2}{|c|}{ DM } \\
\hline \multicolumn{5}{|c|}{ LOG MHR } \\
\hline Model 1 & $1.133(0.878-1.463)$ & 0.337 & $1.886(1.144-3.107)$ & 0.013 \\
\hline Model 2 & $1.070(0.799-1.431)$ & 0.651 & 1.756 (1.030-2.993) & 0.038 \\
\hline Model 3 & $0.876(0.619-1.238)$ & 0.453 & $2.237(1.172-4.270)$ & 0.015 \\
\hline Model 1 & $0.964(0.670-1.388)$ & 0.845 & $0.522(0.262-1.039)$ & 0.064 \\
\hline Model 2 & $0.951(0.636-1.421)$ & 0.805 & $0.541(0.249-1.060)$ & 0.071 \\
\hline Model 3 & $1.351(0.832-2.193)$ & 0.224 & $0.454(0.182-1.133)$ & 0.091 \\
\hline \multicolumn{5}{|c|}{ LDL CHOLESTEROL } \\
\hline Model 1 & $1.211(1.064-1.379)$ & 0.004 & $1.093(0.883-1.352)$ & 0.416 \\
\hline Model 2 & $1.253(1.117-1.404)$ & $<0.001$ & $1.134(0.948-1.357)$ & 0.170 \\
\hline Model 3 & $1.252(1.091-1.435)$ & 0.001 & $1.034(0.803-1.332)$ & 0.795 \\
\hline \multicolumn{5}{|c|}{ APOLIPOPROTEIN A-I } \\
\hline Model 1 & $1.014(0.598-1.719)$ & 0.960 & $0.502(0.205-1.229)$ & 0.131 \\
\hline Model 2 & $1.023(0.569-1.841)$ & 0.939 & $0.558(0.217-1.437)$ & 0.227 \\
\hline Model 3 & $1.322(0.696-2.511)$ & 0.394 & $0.326(0.108-0.977)$ & 0.045 \\
\hline \multicolumn{5}{|c|}{ APOLIPOPROTEIN B } \\
\hline Model 1 & $1.821(1.123-2.953)$ & 0.015 & $1.192(0.541-2.625)$ & 0.662 \\
\hline Model 2 & $2.410(1.455-3.992)$ & 0.001 & $1.726(0.756-3.939)$ & 0.195 \\
\hline Model 3 & $2.225(1.233-4.015)$ & 0.008 & $1.587(0.555-4.540)$ & 0.389 \\
\hline
\end{tabular}

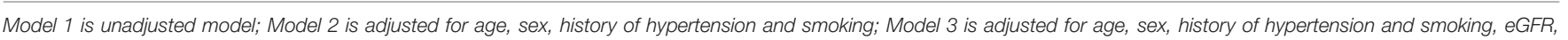
logarithmically transformed levels of C-reactive protein, $\mathrm{HbA1C}$ and triglyceride.

exception of MHR that tended to have a stronger correlation with CIMT ( $r=0.126, p=0.005)$ (Table 2 and Figure 2). After adjustment for confounding risk factors, MHR remained correlated significantly with CIMT in diabetic $(r=0.172$, $p=0.001)$ but not non-diabetic $(r=0.006, p=0.813)$ patients (Table 2). Moreover, we found an upward trend in the distribution of CIMT with increasing quartiles of MHR in both groups (Figure 3). The average (Figure 3A) and maximum CIMT (Figure 3B) in the fourth quartile of MHR $(\geq 13.27)$ were higher than those in the first two quartiles $(<10.13)$ in T2DM patients and those in the first quartile $(<7.52)$ in non-diabetic subjects. Additionally, previous reports demonstrate that men have greater CIMT than women (20), so we sex-stratified our analyses to evaluate the association of MHR with CIMT using linear regression models. After accounting for age in Model 1, MHR was associated with CIMT in males but not in females both in the diabetic and non-diabetic population. After adjustment for other confounding risk factors in Model 2, MHR persisted to be associated with CIMT in males with diabetes but not in those without diabetes (Table 3). 


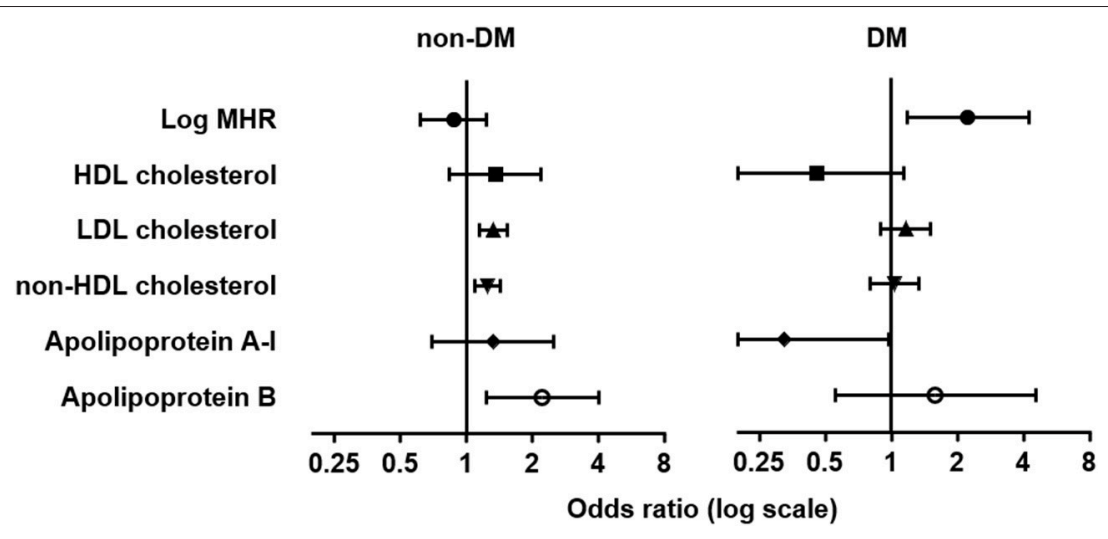

FIGURE 4 | Forest plots of odds ratio (OR) and 95\% confidence interval (Cl) for elevated carotid intima-media thickness. Forest plots were grouped by MHR and traditional lipid variables in the adjusted logistic regression model (model 3) in non-diabetic (non-DM, left) and patients with type 2 diabetes (DM, right). Elevated CIMT was defined as CIMT in the fourth quartile $(\geq 0.73 \mathrm{~mm})$.

\section{Logistic Regression Analyses for Elevated CIMT in Diabetic and Non-diabetic Subjects}

We then analyzed the association between elevated CIMT and different lipid parameters with logistic regression in three models (Table 4). Levels of LDL-C, non-HDL-C, and apoB were associated with elevated CIMT either in univariate analysis (model 1) or after adjusting for age, sex, history of hypertension and smoking (model 2), or with further adjustment for eGFR, log-transformed levels of hsCRP, HbAlc, and triglyceride (model 3 ). However, these associations were markedly attenuated in patients with T2DM. In contrast, we found log-transformed MHR was associated with elevated CIMT either in unadjusted [odds ratio (OR): 1.886, (95\% CI: 1.144-3.107), $p=0.013$ ] or adjusted analyses [OR: 1.756 (95\% CI: 1.030-2.993), $p=0.038$ in model 2; OR: 2.237 (95\% CI: 1.172-4.270), $p=0.015$ in model 3] in diabetic but not non-diabetic subjects (Table 4 and Figure 4).

\section{Study 2}

\section{Baseline Characteristics of the Diabetic Cohort}

Based on these findings from study 1, we propose that MHR is a useful assessment in prediction of subclinical atherosclerosis, as reflected by thickening of CIMT, in patients with T2DM. To evaluate the predictive value of MHR on the progression of CIMT in diabetic patients, 110 diabetic patients with normal CIMT $(<0.73 \mathrm{~mm})$, and a follow-up ultrasonography at 12 months were enrolled in study 2 (Table 5). Among the recruited subjects, $66.4 \%$ patients were male, $30.0 \%$ had smoking habits, and $53.6 \%$ were comorbid with hypertension. The mean age, BMI, HDL$\mathrm{C}$, and LDL-C at baseline was $52.86 \pm 10.32$ years, $25.95 \pm$ $4.64 \mathrm{~kg} / \mathrm{m}^{2}, 1.08 \pm 0.32 \mathrm{mmol} / \mathrm{L}$, and $2.70 \pm 0.92 \mathrm{mmol} / \mathrm{L}$, respectively. The medial basal MHR was 11.00 (IQR: 8.12-13.68). A total of $70 \%$ of patients were on oral hypoglycemic drugs, and $48.2 \%$ were on insulin therapy.

\section{Correlation Between Change in CIMT and Baseline Clinical Variables}

Carotid ultrasonography was performed again at 1 year followup. The mean value of changes in CIMT was $0.037 \mathrm{~mm}$.
Progression of CIMT was detected in 58 (52.7\%) of the subjects. The change in CIMT was positively correlated with count of monocytes $(r=0.350, p<0.001)$ and log-transformed MHR $(r=0.313, p=0.001)$ at baseline (Table 6 and Figure 5). There were also borderline significant associations of change in CIMT with BMI $(r=0.180, p=0.064)$ and log-transformed HbA1C $(r=0.176, p=0.078)$. After adjustment for confounding risk factors including age, sex, BMI, levels of hsCRP, eGFR, HbA1C, LDL-C, and history of hypertension and smoking, there was a positive but non-significant correlation between logtransformed MHR and change in CIMT ( $r=0.223, p=0.079)$. No significant correlation was found between change in CIMT and age, eGFR, log-transformed hsCRP, and traditional lipid parameters (Table 6).

\section{Multivariate Linear Regression Analyses for Change in CIMT}

In multivariate linear regression analysis (Table 7), we found male gender, age, history of hypertension, and baseline CIMT were independently associated with change in CIMT (model I). When log-transformed MHR was included in the model, it remained to be an independent determinant of change in CIMT [ $\beta=0.059$, (95\% CI: 0.013-0.105), $p=0.012$ ] (model II). The inclusion of MHR resulted in an improvement in the predictive ability of the model (change in $R^{2}=0.038, p=0.012$ ). In addition, this association was not affected by controlling for hypoglycemic therapies $[\beta=0.059$, (95\% CI: 0.012-0.105), $p=0.014]$ (model III).

\section{DISCUSSION}

The major findings of the present study are that correlation between MHR and CIMT is enhanced in patients with T2DM than non-diabetic subjects. MHR is superior to traditional lipid variables in association with CIMT thickening and is an independent predictor of the progression of CIMT in patients with T2DM. 
TABLE 5 | Baseline characteristics of the diabetic cohort.

\begin{tabular}{|c|c|}
\hline Number & 110 \\
\hline Male, $n(\%)$ & $73(66.4)$ \\
\hline Age, years & $52.86 \pm 10.32$ \\
\hline Body mass index, kg/m² & $25.95 \pm 4.64$ \\
\hline Smoking, $n(\%)$ & $33(30.0)$ \\
\hline Hypertension, $n(\%)$ & $59(53.6)$ \\
\hline Systolic blood pressure, $\mathrm{mmHg}$ & $133.66 \pm 18.93$ \\
\hline Diastolic blood pressure, $\mathrm{mmHg}$ & $77.34 \pm 12.77$ \\
\hline White blood cell $\left(10^{9} / \mathrm{mL}\right)$ & $6.37 \pm 2.06$ \\
\hline Neutrophil $\left(10^{9} / \mathrm{mL}\right)$ & $3.82 \pm 1.42$ \\
\hline Lymphocyte (109/mL) & $2.02 \pm 0.63$ \\
\hline Monocyte $\left(10^{9} / \mathrm{mL}\right)$ & $0.44 \pm 0.13$ \\
\hline Platelet $\left(10^{9} / \mathrm{mL}\right)$ & $186.38 \pm 53.06$ \\
\hline hsCRP, mg/L & $0.77(0.46-1.59)$ \\
\hline Serum creatinine, $\mu \mathrm{mol} / \mathrm{L}$ & $72.24 \pm 16.60$ \\
\hline Blood urea nitrogen, mmol/L & $5.32 \pm 1.59$ \\
\hline Uric acid, $\mu \mathrm{mol} / \mathrm{L}$ & $330.26 \pm 81.29$ \\
\hline eGFR, mL/min/1.73 m² & $124.06 \pm 22.56$ \\
\hline $\mathrm{HbA1c}, \%$ & $7.60(6.10-9.30)$ \\
\hline Fasting glucose, mmol/L & $7.31(5.65-10.68)$ \\
\hline Postprandial glucose (2h), mmol/L & $15.13(10.46-18.71)$ \\
\hline Fasting insulin, $\mu \mathrm{IU} / \mathrm{mL}$ & $8.45(4.31-15.12)$ \\
\hline Postprandial insulin (2h), $\mu \mathrm{IU} / \mathrm{mL}$ & $38.31(23.39-62.21)$ \\
\hline Triglyceride, $\mathrm{mmol} / \mathrm{L}$ & $1.87(1.19-2.60)$ \\
\hline Total cholesterol, mmol/L & $4.52 \pm 1.16$ \\
\hline HDL cholesterol, mmol/L & $1.08 \pm 0.32$ \\
\hline LDL cholesterol, mmol/L & $2.70 \pm 0.92$ \\
\hline Apolipoprotein A, g/L & $1.28 \pm 0.24$ \\
\hline Apolipoprotein B, g/L & $0.89 \pm 0.25$ \\
\hline MHR & $11.00(8.12-13.68)$ \\
\hline Oral hypoglycemic drugs, $n$ (\%) & $77(70.0)$ \\
\hline Insulin, $n(\%)$ & $53(48.2)$ \\
\hline
\end{tabular}

MHR appears to be a novel and convenient maker with integration of pro-inflammatory and anti-inflammatory indices. In this study, we demonstrate a more prominent role for MHR in prediction of subclinical carotid atherosclerosis in diabetic than non-diabetic populations. First, correlations with CIMT were comparable between $\mathrm{MHR}$ and traditional lipid parameters including HDL-C, LDL-C, non-HDL-C, apoA-I, and apoB in non-diabetic subjects. In patients with T2DM, a stronger correlation was observed between CIMT and MHR, whereas those with other lipid variables tended to be attenuated. Second, we detected an upward trend in the distribution of CIMT with increasing quartiles of MHR in both groups. Especially, CIMT in the fourth quartile of MHR was significantly higher than that in the first two quartiles in diabetic patients, and also higher than that in the fourth quartile of MHR in non-diabetic patients. Third, in the logistic regression analyses, elevated CIMT was independently associated with LDL-C, non-HDL-C, and apoB, whereas these associations were markedly attenuated
TABLE 6 | Correlation between change in CIMT and baseline clinical variables.

\begin{tabular}{lcc}
\hline & \multicolumn{2}{c}{ Chang in CIMT } \\
\cline { 2 - 3 } & $\boldsymbol{r}$ & $\boldsymbol{P}$-value \\
\hline Age & 0.059 & 0.541 \\
BMl & 0.180 & 0.064 \\
Monocytes & 0.350 & $<0.001$ \\
Log hsCRP & 0.055 & 0.621 \\
eGFR & -0.090 & 0.368 \\
Log HbA1C & 0.176 & 0.078 \\
Log triglyceride & -0.004 & 0.968 \\
HDL cholesterol & -0.093 & 0.335 \\
LDL cholesterol & 0.009 & 0.925 \\
non-HDL cholesterol & -0.016 & 0.871 \\
Apolipoprotein A-I & -0.132 & 0.172 \\
Apolipoprotein B & 0.051 & 0.595 \\
Log MHR & 0.313 & 0.001 \\
Log MHR adjusted* & 0.223 & 0.079 \\
\hline
\end{tabular}

*After adjustment for age, sex, history of smoking, hypertension, body mass index, high sensitivity CRP, eGFR, HDA1C, and LDL cholesterol.

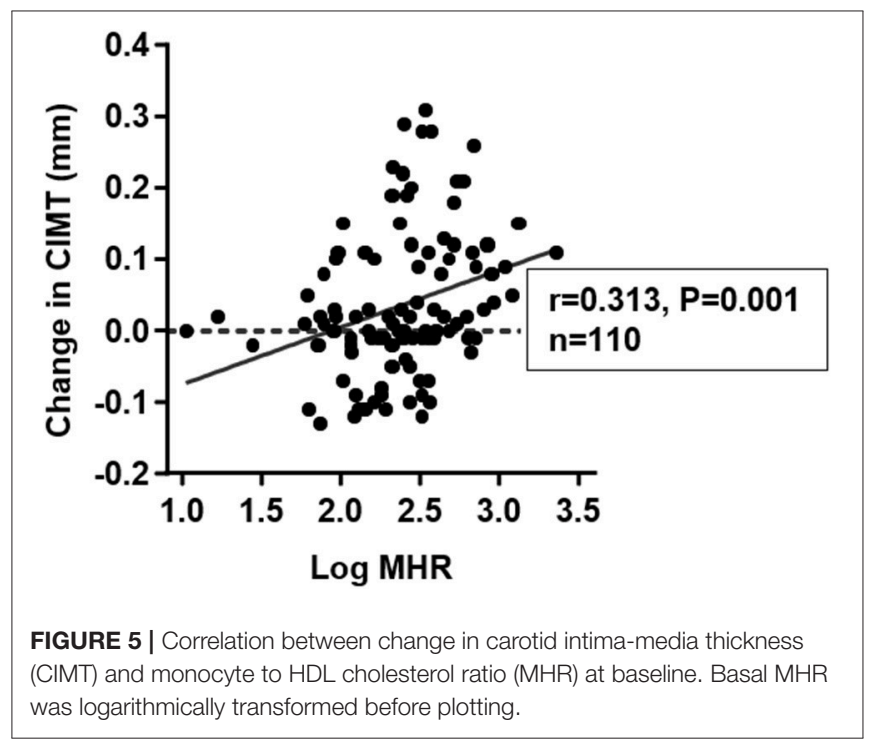

in the diabetic population. Interestingly, we found MHR was independently associated with elevated CIMT both in unadjusted and adjusted models in the diabetic population, while such association was no longer significant in non-diabetic subjects. Finally, in study 2, we further showed that baseline MHR has greater correlation than traditional lipid parameters with change in CIMT at 1 year follow-up.

Monocytes play an important role in the development of diabetic complications (21). Monocyte counts have been shown to be associated with insulin resistance, type 2 diabetes $(22,23)$, coronary artery disease (24), diabetic micro-, and macrovascular complications $(25,26)$. Previously, Matsumura et al. reported that monocyte counts were positively correlated with CIMT in patients with T2DM (10). On the other hand, emerging data suggest that low HDL-C is an important contributor to 


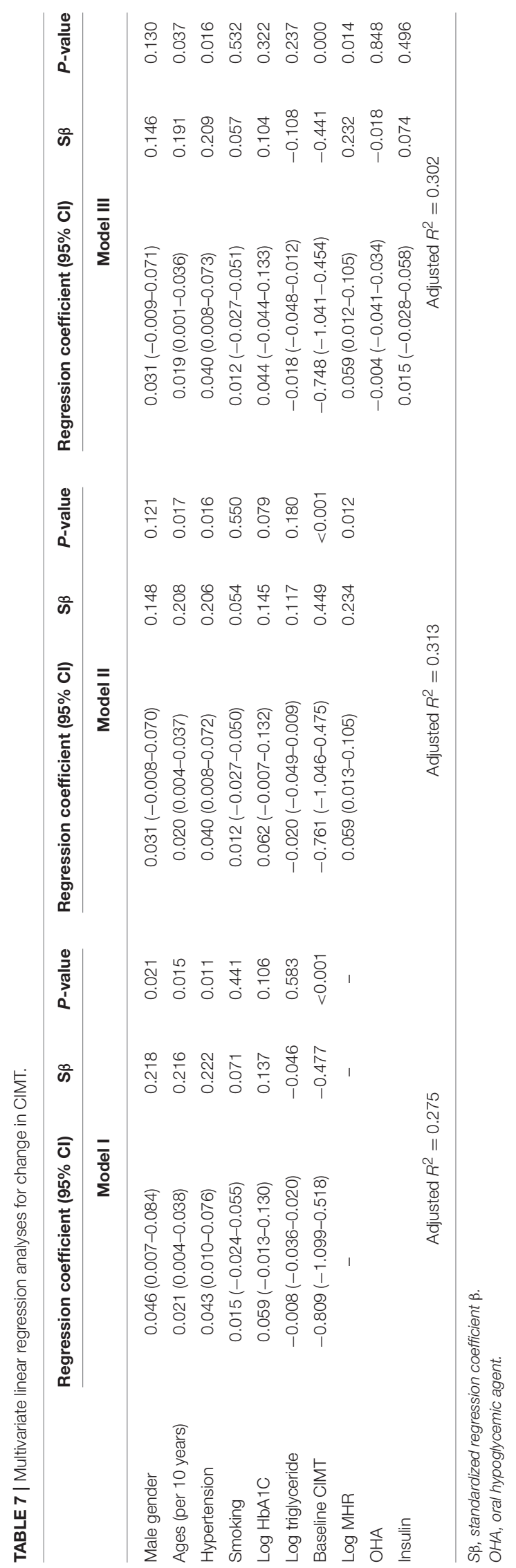

accelerated atherosclerosis in diabetic patients (27). Therefore, the integrated maker, MHR, is supposed to be a better predictive factor than each of the variables in association with vascular structural change in diabetic patients as we demonstrated in the present study. In contrast, the imbalance between pro- and anti-inflammatory mechanisms in non-diabetic conditions is generally relatively moderate, which might explain our findings that MHR is associated with thickening and progression of CIMT in diabetic but not non-diabetic subjects. Previously, Kanbay et al. reported that MHR acts as an independent predictor for cardiovascular events in patients with chronic kidney disease and was increased in parallel with decreasing eGFR (12). MHR was also correlated with flow-mediated dilation of brachial artery in patients with Behcet disease (28). Taken together, these lines of evidence imply that the predictive value of MHR for cardiovascular disease is improved in conditions of chronic inflammatory disorders such as diabetes.

Our data reveal that MHR is both associated with basal thickness and progression of CIMT in patients with T2DM. Noteworthy, while CIMT has been well-evidenced to predict the risk of the subsequent cardiovascular events (29-31), the prognosis value of CIMT rate of change in evaluating cardiovascular risk remain inconclusive (31-34). Findings from the Multi-Ethnic Study of Atherosclerosis (MESA) point to a positive association between CIMT progression and incident stroke (32). The IMPROVE study showed that the fastest maximum CIMT progression, but not other CIMT measures, was significantly associated with the subsequent vascular events (35). Conversely, meta-analyses on 16 cohorts comprised of 36,984 participants in the general population (31), or 21 cohorts comprised of 3,902 participants in patients with T2DM (33), both detected no association between CIMT progression and cardiovascular risk. Nevertheless, the thickening of CIMT, compared to those with unchanged or regressed CIMT, over the 1 year follow-up period appears to some extent reflect the adverse structural changes of the arterial wall. The prognosis value of MHR in predicting CIMT progression awaits examination in prospective trails.

We appreciate limitations in our study. First, this study was a retrospective analysis based on prospectively collected data, and all the enrolled patients were from a single center. Second, the sample size in study 2 was modest and therefore the ability to definitely evaluate the association of CIMT progression with MHR and other variables in patients with T2DM was limited. Third, MHR was not dynamically monitored, so it is still not known changes in MHR is associated with the progression of CIMT. Further prospective studies are warranted to analyze whether decreasing MHR would lead to less progression of atherosclerosis.

\section{CONCLUSIONS}

In summary, this study suggests that elevated MHR is a convenient and effective measure in prediction of the presence and progression of subclinical carotid atherosclerosis in patients with T2DM. 


\section{AUTHOR CONTRIBUTIONS}

JC, LL, and XW study design and conduction, data analysis, and interpretation, manuscript writing. CL, ZL, XS, and YS study conduction, data collection and manuscript revision. FD, RZ, and WS study design and manuscript revision. All authors read and approved the final manuscript.

\section{REFERENCES}

1. Low Wang CC, Hess CN, Hiatt WR, Goldfine AB. Clinical update: cardiovascular disease in diabetes mellitus: atherosclerotic cardiovascular disease and heart failure in type 2 diabetes mellitus - mechanisms, management, and clinical considerations. Circulation. (2016) 133:2459-502. doi: 10.1161/CIRCULATIONAHA.116.022194

2. Martin-Timon I, Sevillano-Collantes C, Segura-Galindo A, Del CanizoGomez FJ. Type 2 diabetes and cardiovascular disease: Have all risk factors the same strength? World J Diabetes. (2014) 5:444-70. doi: 10.4239/wjd.v5.i4.444

3. Mackey RH, Mora S, Bertoni AG, Wassel CL, Carnethon MR, Sibley CT, et al. Lipoprotein particles and incident type 2 diabetes in the multi-ethnic study of atherosclerosis. Diabetes Care. (2015) 38:628-36. doi: 10.2337/dc14-0645

4. Yang W, Li Y, Wang JY, Han R, Wang L. Circulating levels of adipose tissue-derived inflammatory factors in elderly diabetes patients with carotid atherosclerosis: a retrospective study. Cardiovasc Diabetol. (2018) 17:75. doi: 10.1186/s12933-018-0723-y

5. Creager MA, Luscher TF, Cosentino F, Beckman JA. Diabetes and vascular disease: pathophysiology, clinical consequences, and medical therapy: part I. Circulation. (2003) 108:1527-32. doi: 10.1161/01.CIR.0000091257.27563.32

6. Hilgendorf I, Swirski FK, Robbins CS. Monocyte fate in atherosclerosis. Arterioscler Thromb Vasc Biol. (2015) 35:272-9. doi: 10.1161/ATVBAHA.114.303565

7. Libby P. Inflammation in atherosclerosis. Arterioscler Thromb Vasc Biol. (2012) 32:2045-51. doi: 10.1161/ATVBAHA.108.179705

8. Moore KJ, Sheedy FJ, Fisher EA. Macrophages in atherosclerosis: a dynamic balance. Nat Rev Immunol. (2013) 13:709-21. doi: 10.1038/ nri3520

9. Chapman CM, Beilby JP, McQuillan BM, Thompson PL, Hung J. Monocyte count, but not C-reactive protein or interleukin-6, is an independent risk marker for subclinical carotid atherosclerosis. Stroke. (2004) 35:1619-24. doi: 10.1161/01.STR.0000130857.19423.ad

10. Matsumura T, Taketa K, Motoshima H, Senokuchi T, Ishii N, Kinoshita H, et al. Association between circulating leukocyte subtype counts and carotid intima-media thickness in Japanese subjects with type 2 diabetes. Cardiovasc Diabetol. (2013) 12:177. doi: 10.1186/1475-2840-12-177

11. Yilmaz M, Kayancicek H. A new inflammatory marker: elevated monocyte to HDL cholesterol ratio associated with smoking. J Clin Med. (2018) 7:76. doi: $10.3390 / j \mathrm{~cm} 7040076$

12. Kanbay M, Solak Y, Unal HU, Kurt YG, Gok M, Cetinkaya H, et al. Monocyte count/HDL cholesterol ratio and cardiovascular events in patients with chronic kidney disease. Int Urol Nephrol. (2014) 46:1619-25. doi: 10.1007/s11255-014-0730-1

13. Wei XB, Chen F, Huang JL, He PC, Wei YX, Tan N, et al. Novel risk biomarker for infective endocarditis patients with normal left ventricular ejection fraction- monocyte to high-density lipoprotein cholesterol ratio. Circ J. (2017) 82:283-8. doi: 10.1253/circj.CJ-17-0427

14. Geroulakos GA, O'gorman D, Kalodiki E, Sheridan D, Nicolaides A. The carotid intima-media thickness as a marker of the presence of severe symptomatic coronary artery disease. Eur Heart J. (1994) 15:781-5. doi: 10.1093/oxfordjournals.eurheartj.a060585

15. Bots M, Hoes A, Koudstaal PJ, Hofman A, Grobbee D. Common carotid intima-media thickness and risk of stroke and myocardial infarction: the Rotterdam Study. Circulation. (1997) 96:1432-7. doi: 10.1161/01.CIR.96.5.1432

16. American Diabetes A. Standards of medical care in diabetes-2012. Diabetes Care. (2012) 35 (Suppl 1):S11-63. doi: 10.2337/dc12-s011

\section{FUNDING}

This study was supported by National Natural Science Foundation of China (Grant No. 81670451, 81470469, 81770430, 81870179), Shanghai Rising-Star Program (Grant No. 17QA1403000), Shanghai Municipal Commission of Health and Family Planning (Grant No. 2018YQ17).

17. Chobanian AV, Bakris GL, Black HR, Cushman WC, Green LA, Izzo JL Jr, et al. The seventh report of the joint national committee on prevention, detection, evaluation, and treatment of high blood pressure: the JNC 7 report. JAMA. (2003) 289:2560-71. doi: 10.1001/jama.289.19.2560

18. Grundy SM, Cleeman JI, Merz CN, Brewer HB Jr, Clark LT, Hunninghake DB, et al. Implications of recent clinical trials for the national cholesterol education program adult treatment panel III guidelines. Circulation. (2004) 110:227-39. doi: 10.1161/01.CIR.0000133317.49796.0E

19. Levey AS, Stevens LA, Schmid CH, Zhang YL, Castro AF III, Feldman HI, et al. A new equation to estimate glomerular filtration rate. Ann Internal Med. (2009) 150:604-12. doi: 10.7326/0003-4819-150-9-200905050-00006

20. Juonala M, Kahonen M, Laitinen T, Hutri-Kahonen N, Jokinen E, Taittonen L, et al. Effect of age and sex on carotid intima-media thickness, elasticity and brachial endothelial function in healthy adults: the cardiovascular risk in Young Finns Study. Eur Heart J. (2008) 29:1198-206. doi: 10.1093/eurheartj/ehm556

21. Williams MD, Nadler JL. Inflammatory mechanisms of diabetic complications. Curr Diabetes Rep. (2007) 7:242-8. doi: 10.1007/s11892-007-0038-y

22. Ohshita K, Yamane K, Hanafusa M, Mori H, Mito K, Okubo M, et al. Elevated white blood cell count in subjects with impaired glucose tolerance. Diabetes Care. (2004) 27:491-6. doi: 10.2337/diacare.27.2.491

23. Vozarova B, Weyer C, Lindsay RS, Pratley RE, Bogardus C, Tataranni PA. High white blood cell count is associated with a worsening of insulin sensitivity and predicts the development of type 2 diabetes. Diabetes. (2002) 51:455-61. doi: 10.2337/diabetes.51.2.455

24. Kannel WB, Anderson K, Wilson PW. White blood cell count and cardiovascular disease. Insights from the Framingham Study. JAMA. (1992) 267:1253-6. doi: 10.1001/jama.1992.03480090101035

25. Tong PC, Lee KF, So WY, Ng MH, Chan WB, Lo MK, et al. White blood cell count is associated with macro- and microvascular complications in chinese patients with type 2 diabetes. Diabetes Care. (2004) 27:216-22. doi: $10.2337 /$ diacare.27.1.216

26. Cavalot F, Massucco P, Perna P, Traversa M, Anfossi G, Trovati M. White blood cell count is positively correlated with albumin excretion rate in subjects with type 2 diabetes. Diabetes Care. (2002) 25:2354-5. doi: $10.2337 /$ diacare.25.12.2354-a

27. Expert Panel on Detection, Evaluation, and Treatment of High Blood Cholesterol in Adults. Executive summary of the third report of the national cholesterol education program (NCEP) expert panel on detection, evaluation, and treatment of high blood cholesterol in adults (Adult Treatment Panel III). JAMA. (2001) 285:2486-97. doi: 10.1001/jama.285.19.2486

28. Acikgoz N, Kurtoglu E, Yagmur J, Kapicioglu Y, Cansel M, Ermis N. Elevated monocyte to high-density lipoprotein cholesterol ratio and endothelial dysfunction in behcet disease. Angiology. (2018) 69:65-70. doi: 10.1177/0003319717704748

29. Lorenz MW, Markus HS, Bots ML, Rosvall M, Sitzer M. Prediction of clinical cardiovascular events with carotid intima-media thickness: a systematic review and meta-analysis. Circulation. (2007) 115:459-67. doi: 10.1161/CIRCULATIONAHA.106.628875

30. Baldassarre D, Hamsten A, Veglia F, de Faire U, Humphries SE, Smit AJ, et al. Measurements of carotid intima-media thickness and of interadventitia common carotid diameter improve prediction of cardiovascular events: results of the IMPROVE (Carotid Intima Media Thickness [IMT] and IMT-Progression as Predictors of Vascular Events in a High Risk European Population) study. J Am Coll Cardiol. (2012) 60:1489-99. doi: $10.1016 /$ j.jacc.2012.06.034 
31. Lorenz MW, Polak JF, Kavousi M, Mathiesen EB, Volzke H, Tuomainen TP, et al. Carotid intima-media thickness progression to predict cardiovascular events in the general population (the PROG-IMT collaborative project): a meta-analysis of individual participant data. Lancet. (2012) 379:2053-62. doi: 10.1016/S0140-6736(12)60441-3

32. Polak JF, Pencina MJ, O’Leary DH, D'Agostino RB. Common carotid artery intima-media thickness progression as a predictor of stroke in multi-ethnic study of atherosclerosis. Stroke. (2011) 42:3017-21. doi: 10.1161/STROKEAHA.111.625186

33. Lorenz MW, Price JF, Robertson C, Bots ML, Polak JF, Poppert H, et al. Carotid intima-media thickness progression and risk of vascular events in people with diabetes: results from the PROG-IMT collaboration. Diabetes Care. (2015) 38:1921-9. doi: 10.2337/dc14-2732

34. Hirano M, Nakamura T, Kitta Y, Takishima I, Deyama J, Kobayashi $\mathrm{T}$, et al. Short-term progression of maximum intima-media thickness of carotid plaque is associated with future coronary events in patients with coronary artery disease. Atherosclerosis. (2011) 215:507-12. doi: 10.1016/j.atherosclerosis.2011.01.014
35. Baldassarre D, Veglia F, Hamsten A, Humphries SE, Rauramaa R, de Faire U, et al. Progression of carotid intima-media thickness as predictor of vascular events: results from the IMPROVE study. Arterioscler Thromb Vasc Biol. (2013) 33:2273-9. doi: 10.1161/ATVBAHA.113. 301844

Conflict of Interest Statement: The authors declare that the research was conducted in the absence of any commercial or financial relationships that could be construed as a potential conflict of interest.

Copyright (C) 2019 Chen, Li, Liu, Shen, Ding, Shu, Zhang, Shen, Lu and Wang. This is an open-access article distributed under the terms of the Creative Commons Attribution License (CC BY). The use, distribution or reproduction in other forums is permitted, provided the original author(s) and the copyright owner(s) are credited and that the original publication in this journal is cited, in accordance with accepted academic practice. No use, distribution or reproduction is permitted which does not comply with these terms. 